\title{
Acceleration of block-matching algorithms using a custom instruction-based paradigm on a Nios II microprocessor
}

\author{
Diego González, Guillermo Botella, Carlos García, Manuel Prieto and Francisco Tirado
}

\begin{abstract}
This contribution focuses on the optimization of matching-based motion estimation algorithms widely used for video coding standards using an Altera custom instruction-based paradigm and a combination of synchronous dynamic random access memory (SDRAM) with on-chip memory in Nios II processors. A complete profile of the algorithms is achieved before the optimization, which locates code leaks, and afterward, creates a custom instruction set, which is then added to the specific design, enhancing the original system. As well, every possible memory combination between on-chip memory and SDRAM has been tested to achieve the best performance. The final throughput of the complete designs are shown. This manuscript outlines a low-cost system, mapped using very large scale integration technology, which accelerates software algorithms by converting them into custom hardware logic blocks and showing the best combination between on-chip memory and SDRAM for the Nios 11 processor.
\end{abstract}

Keyword: Computer vision, Optical flow, MPEG compression, Block-matching algorithm, Nios II, FPGA, Custom instructions, Embedded systems

\section{Introduction}

Real-time motion estimation is an important task to be computed using machine vision technology and a multimedia scope. For example, one of the most timeconsuming issues when computing standards with video coding and transmission has to do with the ubiquitous portable consumer electronic devices, all with multimedia capabilities, that require the efficient implementation of video coding algorithms, creating a trade-off between accuracy, efficiency, and power consumption. There is a profusion of motion estimation algorithms and systems; many of them are frequently used in multimedia tasks and video coding standards, such as motion compensation and coding $[1,2]$.

When considering motion estimation for multimedia purposes, the main point is to avoid the use of temporal redundancy of video data for storage and transmission [2,3]. Motion estimation for multimedia coding is achieved mostly through block-matching techniques

\footnotetext{
* Correspondence: dgonzalez@grupobme.es

Department of Computer Architecture and Automation, Complutense University, Ciudad Universitaria s/nMadrid 28040, Spain
}

[4-8] that analyze the macro blocks (blocks of pixels, commonly called MBs) of the reference frame in order to estimate the closest block to the one in the current frame. Accordingly, the motion vector is defined as an offset from the current frame of $\mathrm{MB}$ coordinates to the $\mathrm{MB}$ coordinates in the reference frame. An overview of the process is shown in Figure 1.

This method of coding the processed frame with motion estimation using video is also known as inter-frame. There are several previous works regarding motion estimation hardware acceleration [9-11] and, specifically, block-matching algorithms [12], though none of them explore the custom instruction paradigm. Looking into block-matching techniques, three frequently used techniques can be classified: the full-search technique (FST) [4], the three-step-search technique (TSST) [13], and the two-dimensional logarithmic-search technique (2DLOG) [14].

The FST [4] matches all possible blocks within a search window in the reference frame to determine the closest block to the one fixed in the current frame (Figure 2). The closest is the one with the minimum 


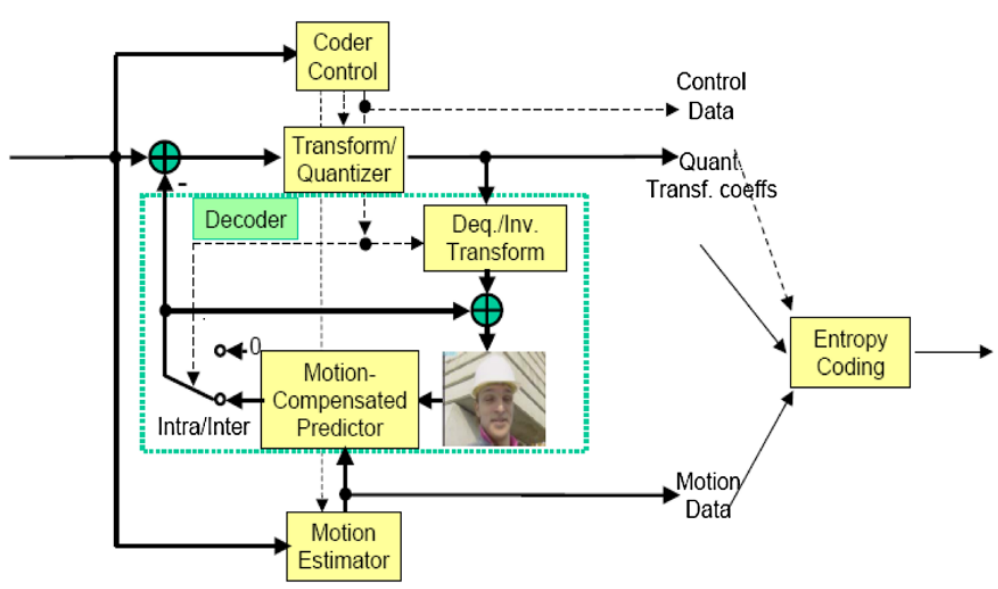

Figure 1 Motivation part of the MPEG-4 scheme.

summation of absolute differences (SAD), which is defined as:

$$
S A D(x, y ; u, v)=\sum_{x=0}^{31} \sum_{y=0}^{31}\left|I_{t}(x, y)-I_{t-1}(x+u, y+v)\right|
$$

where $I_{t}(x, y)$ represents the pixel value at the coordinate $(x, y)$ in the frame $t$, and the $(u, v)$ is the displacement of the candidate MB. For example, for a $32 \times 32$ block, the FST algorithm requires 1,024 subtractions and 1,023 additions to calculate a SAD. The required number of checking blocks is $(1+2 \mathrm{~d})^{2}$, while the search window is limited within $\pm \mathrm{d}$ pixels, and currently, a power of two is used for it.

The TSST $[13,15,16]$ selects nine candidate points, including the center point and eight checkpoints on the boundary of the center movement ratio search, fast forwards to the matching point with the minimum SAD, and reduces the step size by half in each of its three steps (Figure 3). The final step stops the search process with the optimal MV so the minimum SAD can be obtained.

The 2DLOG [14] uses a pattern cross search (+) for each step until the step size is one pixel, with the initial step size being $d / 4$. The step size is reduced by half only when the minimum point of the previous step is at the center or the current minimum point reaches the search window boundary (Figure 4). If none of these two conditions is accomplished, the step size remains the same.

The organization of the paper is as follows: in Section 2, a Nios II processor overview is presented. Section 2.1 outlines the custom instruction types. Section 2.2 discusses the different memory architectures. Section 3 shows the methodology used in this work. Section 4 shows and discusses the results from the different designs. Conclusions are presented in Section 5.

\section{Nios II processor}

Nios II $[17,18]$ is a 32 -bit general soft-core embedded processor, which allows the acceleration of time-critical software algorithms by adding custom instructions to its instruction set. It is part of a three-member family, fast, economy, or standard, each one optimized for a specific price and performance range.

The Nios II/f Fast central processing unit (CPU) is optimized for maximum performance $[19,20]$; it delivers up to 220 DMIPs of performance in the Stratix II family of field programmable gate arrays (FPGAs), placing it squarely in the advanced RISC machine (ARM) 9 [21] class of processors. This performance can be fitted to

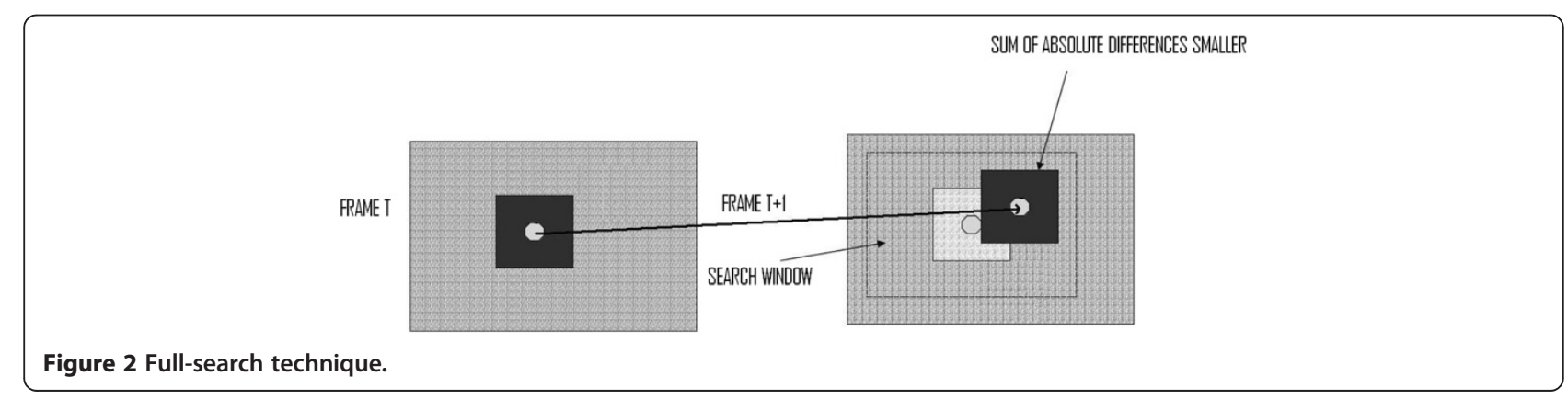




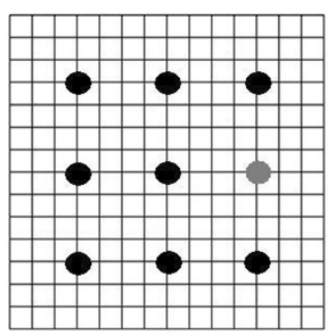

(A)

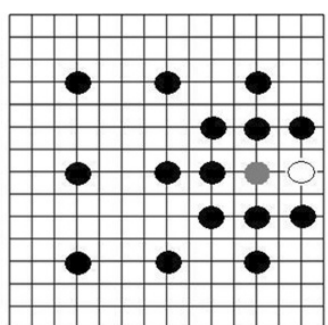

(B)

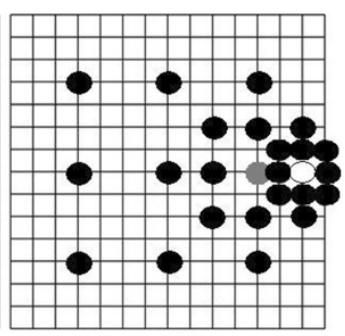

(C)

Figure 3 Three-step-search technique: (A) first step, (B) second step, (C) third step.

meet constraints using custom instructions, highbandwidth switch fabric, and hardware accelerators. It supports fixed and variable cycle operations. The Nios II/e Economy CPU is optimized for the lowest cost, resulting in a smaller FPGA footprint. The Nios II/s Standard CPU delivers over 120 DMIPs while consuming only 930 LEs (Stratix II [22]), creating a balance between processing performance and logic element usage.

\subsection{Nios II custom instructions}

A better design can be achieved with the Nios II custom instruction-based paradigm [23] by designing custom logic blocks adjacent to the arithmetic logic unit (ALU) in the processor's datapath (Figure 5) thus allowing the designer to reduce a complex sequence of standard instructions to a single instruction implemented in hardware. The Nios II processor uses GNU compiler collection (GCC) built-in functions to map custom instructions [24]; therefore, it is feasible to use macro directly in the $\mathrm{C}$ or $\mathrm{C}++$ application code, avoiding having to write assembly code to access the custom instructions. The Nios II processor supports different types of custom instructions. Figure 6 lists the additional ports

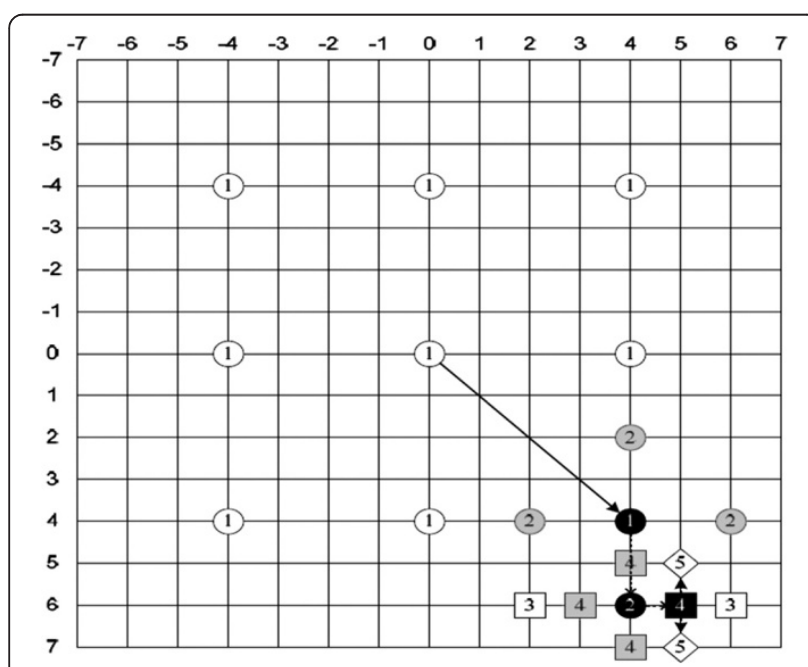

Figure 4 The search path of the 2DLOG search algorithm. that accommodate the different custom instruction types, where only the ports used for the specific custom instruction implementation are required.

There are four available types of custom instructions that can be used to meet each application's constraint and requirements.

The combinational type of custom instruction consists of a logic block that completes its logic function in a single clock cycle. The multi-cycle (or sequential) type of custom instruction consists of a logic block that requires two or more clock cycles to complete an operation. An extended type of custom instruction allows a single custom logic block to implement several different operations using an index to specify which operation the logic block will have to perform. The internal register file custom instructions allow access to its own internal register file, providing the flexibility to specify whether the custom instruction reads its operands from the Nios II processor's register file or from the custom instruction's own internal register file.

\subsection{Memory system design for machine vision implementation}

Initially, the design was implemented without using a custom instruction-based paradigm; instead, the memory types were managed in order to reach a better combination to achieve a faster design. According to the Nios II specifications [17], there are four types of memory that could be used in the Nios II processor-based design: onchip memory, external static random access memory (SRAM), flash memory, and synchronous dynamic random access memory (SDRAM). Here is a brief analysis of the advantages and disadvantages of each:

\section{On-chip memory}

On-chip memory is connected to the circuit board without using any external connection, because it is embedded inside the FPGA. As an advantage, this is the fastest type of memory that can be used in an FPGA-based embedded system. It allows for the pipelining of transactions, and it does not require additional circuit-board wiring, which translates to a very low cost. A disadvantage is that 


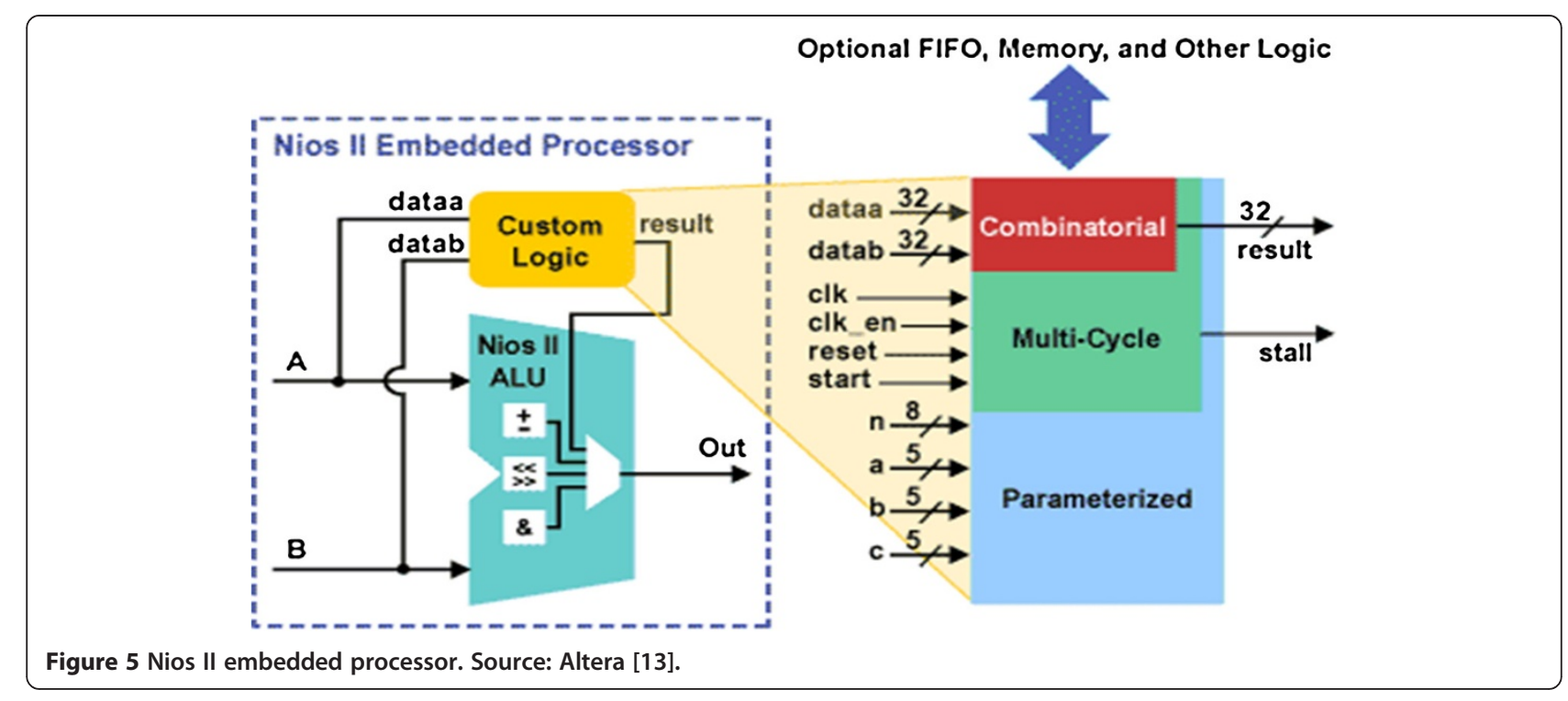

it raises the volatility, which makes it lose its contents when power is disconnected, and it has limited capacity, because designed memory capacity depends only on the specific FPGA device. Considering the advantages and disadvantages, on-chip memories are mainly utilized for storing boot code or look-up tables (LUT).

\section{External RAM}

SRAM is implemented outside of FPGA and connected to it by a shared and simple bidirectional bus. As an advantage, the throughput remains high, though still lower than on-chip memories, but the storage capacity is larger. However, they are more expensive per MByte than other high-capacity memory types, such as SDRAM, and they consume more board space per MByte than both SDRAM and FPGA on-chip memory, which almost consumes none.

\section{Flash memory}

Flash memory is a non-volatile memory type external to the FPGA, since FPGAs do not contain it. It has several advantages: it retains the data after the power is off; and

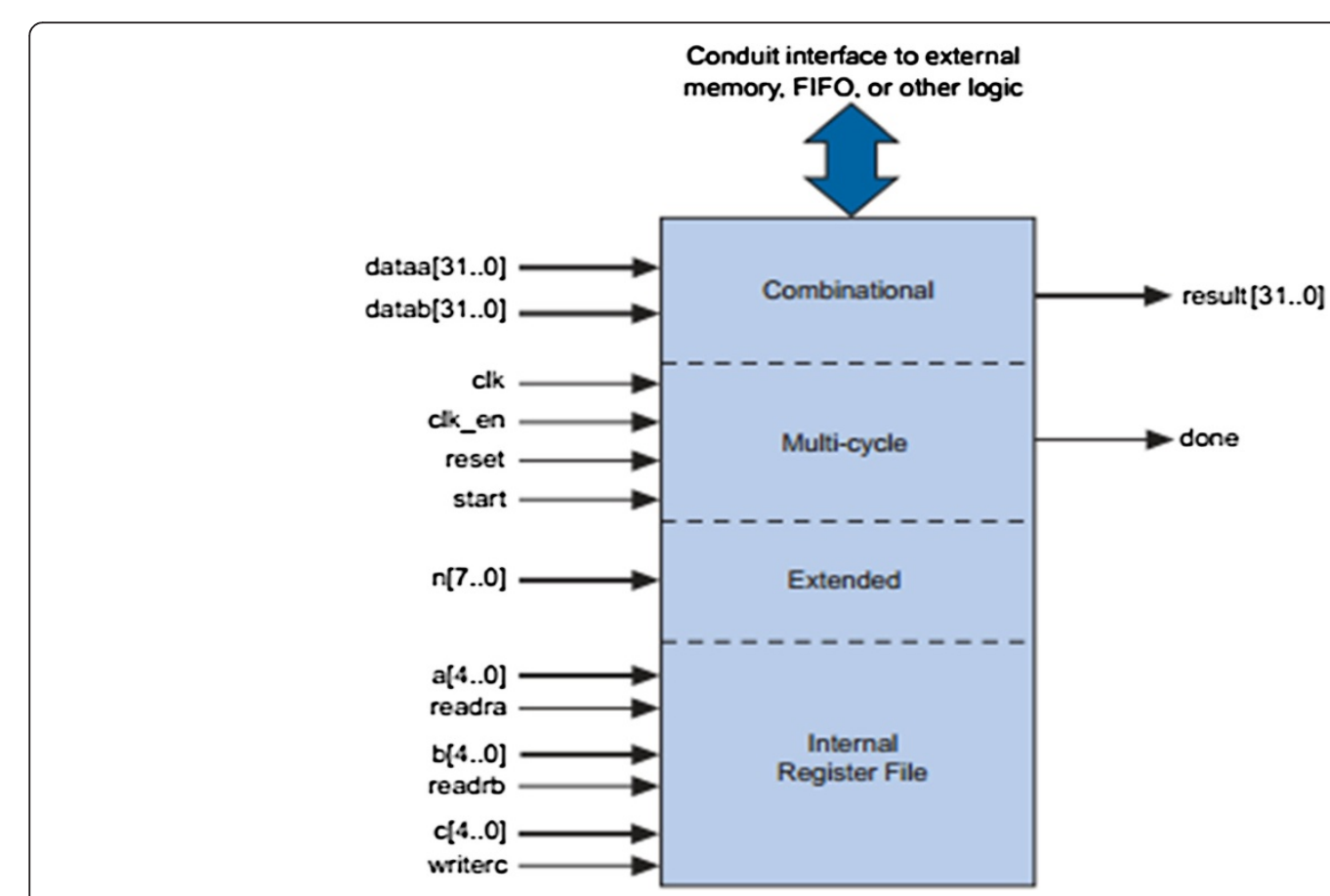

Figure 6 Custom instructions types. Source: Altera [15]. 


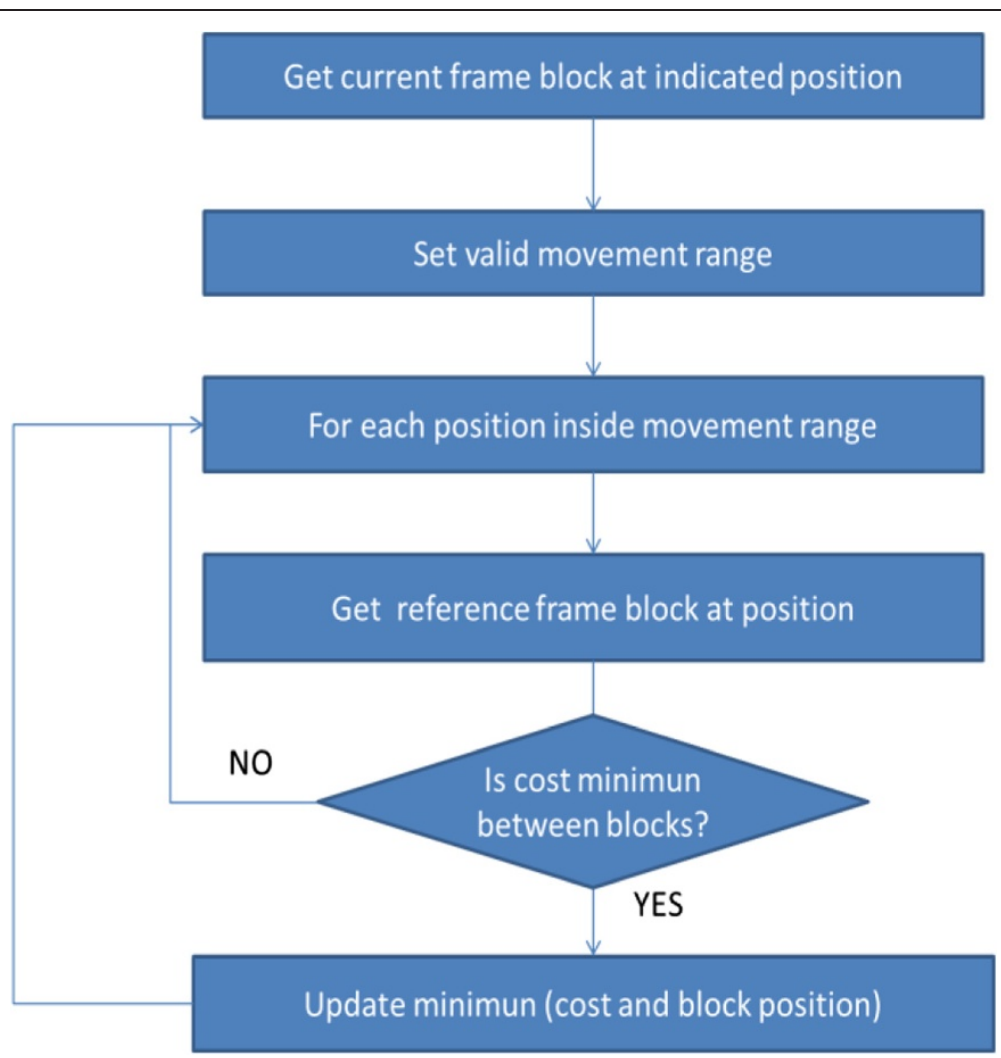

Figure 7 FST flow chart.

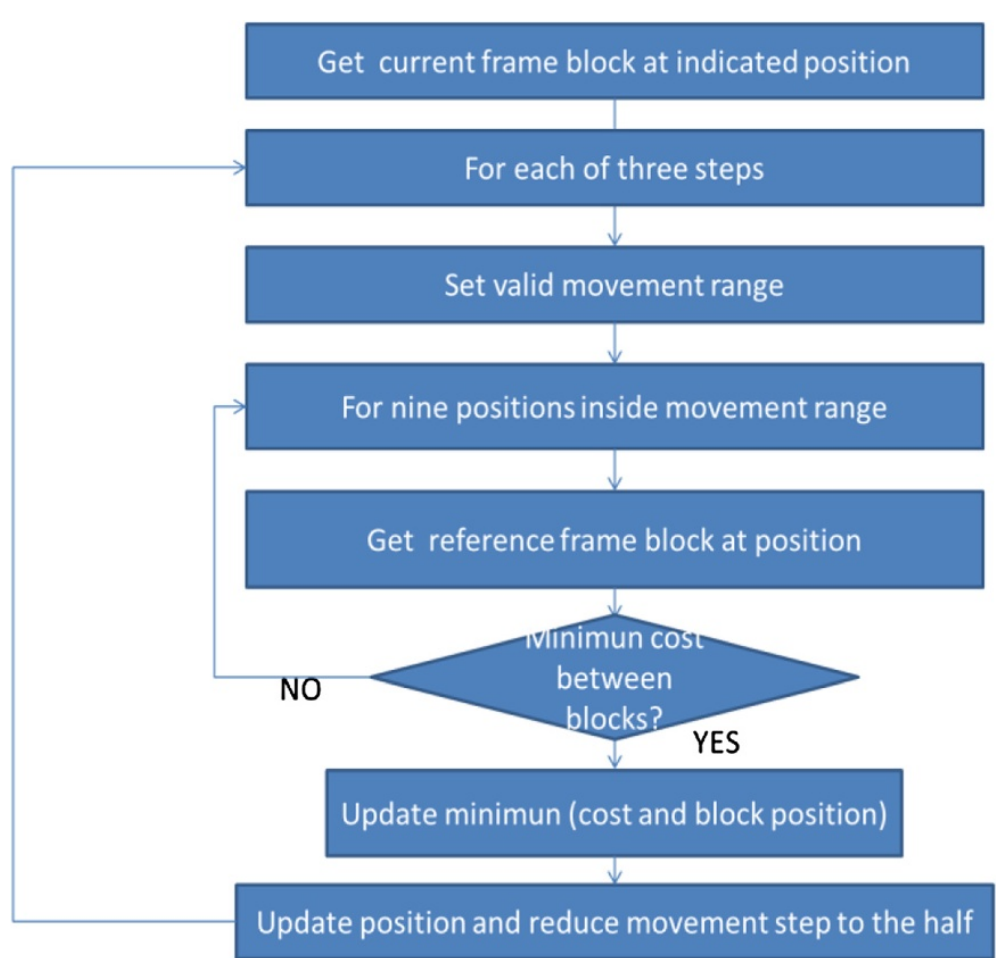

Figure 8 TSST flow chart. 


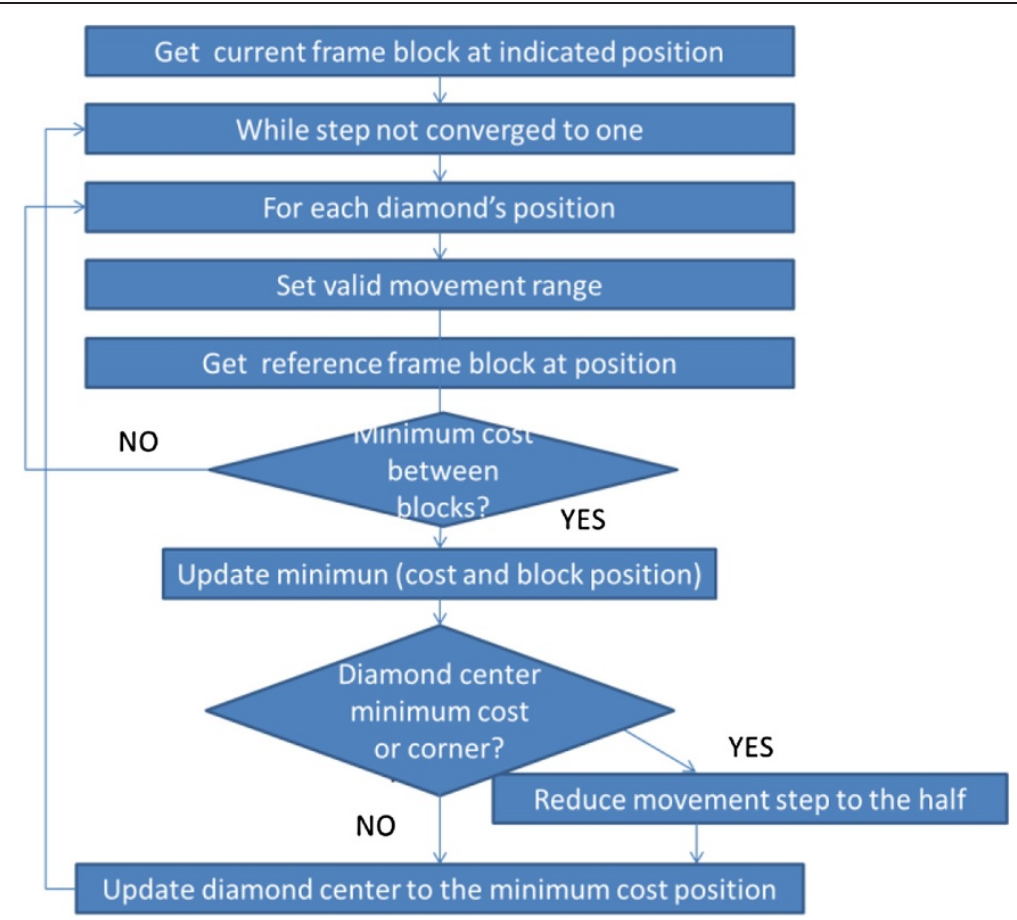

Figure 9 2DLOG flow chart.

it is low cost, erasable, and durable. One disadvantage is that it has a very high writing latency, due to the writing process requesting specific commands and bus transactions. Moreover, before flash memory can be written, it must be erased, and since individual words cannot be erased, entire sections of the flash must be erased as a unit. Because of their advantages and disadvantages, flash memories are mainly used to hold microprocessor boot code as well as any data, which need to be preserved in the case of a power failure.

\section{SDRAM}

SDRAM is another type of volatile memory. It organizes the memory space in columns, rows, and banks. It is similar to SRAM, but it must be refreshed periodically to keep its data. It requires one specific hardware controller, which drives the timing, address multiplexing, and refreshes every cycle. Its advantages are that it is low cost and it has a large capacity. Moreover, its power consumption is lower than SRAM. As a drawback, its required SDRAM controller occupies a major part of the interface. SDRAM latency is always greater than that of regular external SRAM or FPGA on-chip memory, although some types of SDRAM can achieve higher clock frequencies than SRAM. Due to its advantages and drawbacks, the devices that work with SDRAM are usually low cost and high capacity.

From these four types of memory, two of them were used here. Flash memory was discarded as an option due to its high latency and low capacity; additionally,

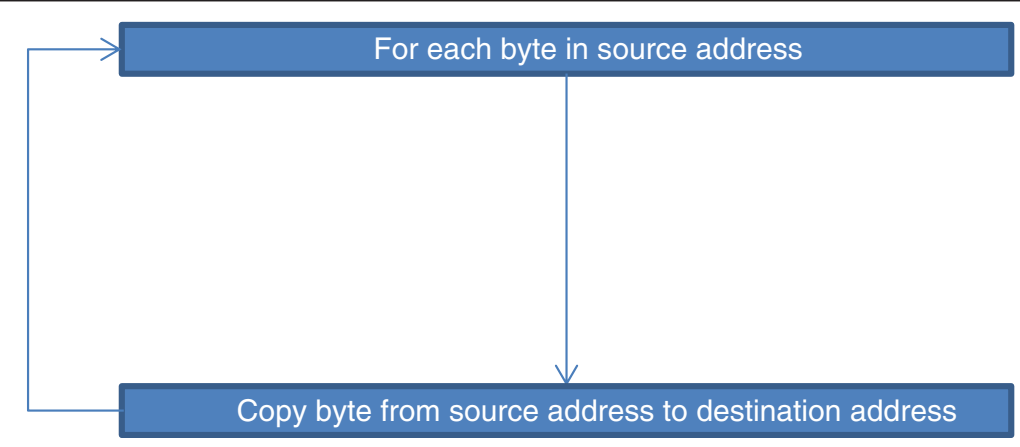

Figure 10 CopyBlock flow chart. 


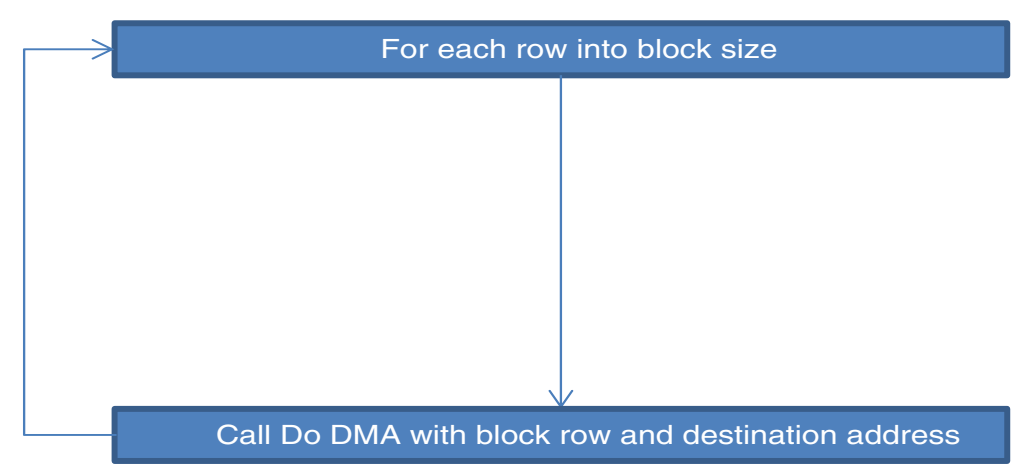

Figure 11 GetBlock flow chart.

non-volatile memory was not needed for this design. SRAM was discarded because of its cost per MByte, which is more expensive than SDRAM, and designing a low-cost system was of prime importance. On-chip memory was utilized, because it is the fastest memory type available on the FPGA, and it is low cost. SDRAM was also used in the design, because of its low cost and very high capacity; moreover, we needed another memory, apart from the on-chip, for allocating the entire project.

\section{Methodology}

The methodology follows in two sections: the first section presents the parameters for the achieved designs through the use of Nios II custom instructions, and the second section presents the improvement in terms of throughput achieved through every valid and possible combinations of the on-chip and SDRAM memory into a design using the Nios II processor.

\subsection{Nios II custom instructions}

Once the different custom instruction types and the advantages and disadvantages of each one were determined, a profile of the three presented algorithms was made using the well-known code blocks tool [25]. This allowed facing up directly to the time leak point, where better performance improvement could be achieved to replace source code functions for custom instructions. For a better comprehension of the profiling, flow charts are provided in Figures 7, 8, and 9 for FST [4], TSST $[13,15,16]$, and 2DLOG [14], respectively.

Figures 10, 11, and 12 show CopyBlock, GetBlock, and GetCost functions, respectively.

Tables 1, 2, and 3 show the profiling results accomplished for complete executions of the motion estimation process, highlighting FST [4], TSST $[13,15,16]$, and 2DLOG [14], accordingly.

By examining the profiling results, some conclusions can be made about the most appropriate part of the

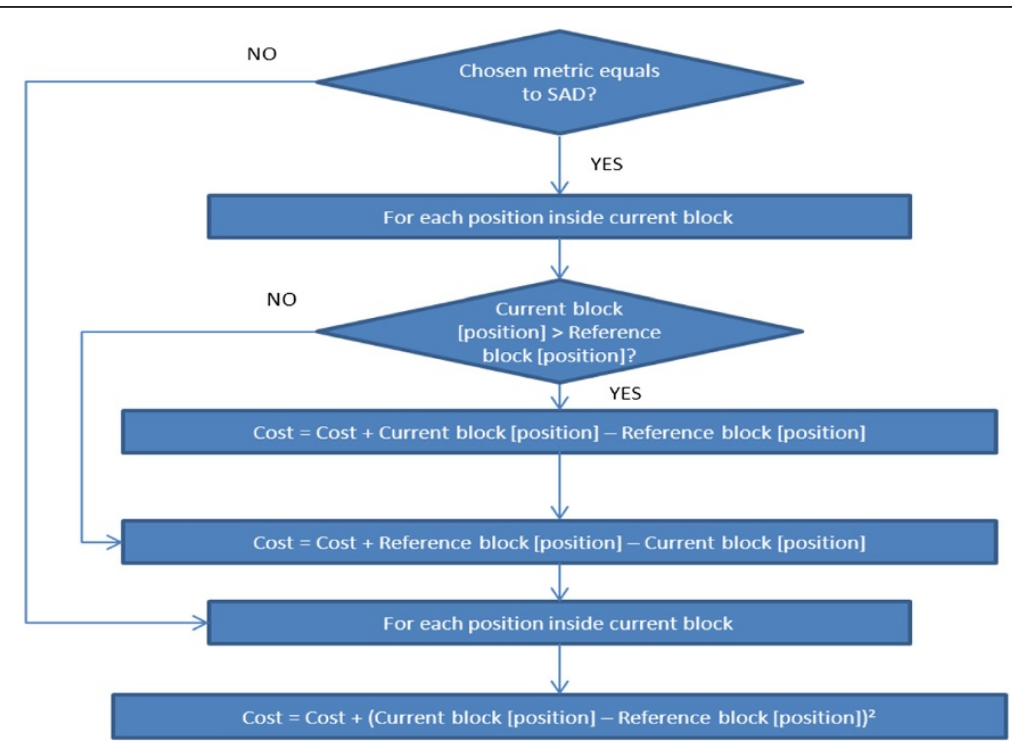

Figure 12 GetCost flow chart. 
Table 1 FST [4] algorithm profiling

\begin{tabular}{lcc}
\hline Function (FST) & \% Time & Calls \\
\hline CopyBlock & 8.57 & 4844640 \\
GetBlock & 0.48 & 302790 \\
GetCost & 90.95 & 302691 \\
FST & approximately 0 & 99 \\
\hline
\end{tabular}

source code to be replaced by a specific custom instruction.

The GetCost function was the first focus; moreover, as was shown in the previous tables, almost all the execution time was taken. Regarding the custom instruction types, their weaknesses, and the GetCost function source code structure, the best approach was clearly a combinatorial custom instruction, due to its speed (only one clock cycle). Although, at first glance, it might seem a multi-cycle custom instruction should be utilized; this was impossible due to the necessary accumulated result in calculating the accumulated SAD between each pair of pixels from the selected blocks. The extended custom instruction was discarded because only one kind of operation was needed between each pair of pixels (calculate SAD). The internal register file custom instruction extends from the multi-cycle one, and that one was discarded for reasons previously discussed. The best candidate was clearly monocycle custom instruction, because of its low latency (only one cycle) and its easiness.

After applying the specific custom instruction used, the performance enhancement for every video-coding motion estimation algorithm in two different video sequences is presented. Additionally, the size of each window $(8,16,32$ pixels), previously explained in Figure 2, was analyzed using every possible combination with a MB size of 16, 32, 64 pixels, respectively. As well, every Nios II processor type was used in our experiments. All results for the Foreman test sequence [26] are shown in Figure 13, and all the results for the Carphone test sequence [26] are shown in Figure 14. In Tables 4 and 5, respectively, the time spent executing time reduction for the presented results is shown for the two video sequences for the improvement achieved for the Foreman and Carphone test benches [26].

Improvements were not dependent on the window search size; results only depended on the size of the macro block processor and algorithm; lower improvement

Table 2 TSST $[13,15,16]$ algorithm profiling

\begin{tabular}{lcc}
\hline Function (TSST) & \% Time & Calls \\
\hline CopyBlock & approximately 0 & 38832 \\
GetBlock & approximately 0 & 2427 \\
GetCost & approximately 100 & 2328 \\
TSST & approximately 0 & 99 \\
\hline
\end{tabular}

Table 3 2DLOG [14] algorithm profiling

\begin{tabular}{lcc}
\hline Function (2DLOG) & \% Time & Calls \\
\hline CopyBlock & approximately 0 & 43280 \\
GetBlock & approximately 0 & 2705 \\
GetCost & approximately 100 & 2606 \\
TSST & approximately 0 & 99 \\
\hline
\end{tabular}

correlated with a higher size. For example, for the FST algorithm, performances moved from $10 \%$ to $55 \%$, while for the other algorithms that were different than the pure exhaustive full search (TSST and 2DLOG), the performance moved from $30 \%$ to $0 \%$.

Regarding the FST algorithm, a noteworthy saving of execution time was shown; however, looking inside each processor, the greatest improvement was seen with the Nios II/e processor, around $40 \%$ of saved execution time, independent of the window search using macro block sizes of 16 and 32 pixels, although using a macro block size of 64 pixels was around 30\%. Using the Nios II/s processor, the percentage of saved execution time was reduced around 20\%, more or less in half; but using the Nios II/f processor, the maximum saved execution time was obtained with FST, around $45 \%$ off, which was reduced when using a macro block size of 64 pixels to $10 \%$.

Regarding 2DLOG, a noteworthy improvement in execution time was shown, around 35\%, using the Nios II/e processor using macro block sizes of 16 and 32 pixels, although using a macro block size of 64 pixels was around 20\%. By using the Nios II/s processor, the saving was more or less $10 \%$, which is not much compared with the saving achieved with the FST algorithm. Compared with the Nios II/f processor, the improvement was still less, around 5\%.

Finally, regarding the TSST algorithm, the table shows great improvement using the Nios II/e processor, saving around $35 \%$, as with the 2DLOG algorithm, but using the Nios II/s processor, an improvement was achieved of around $10 \%$ of the spent execution time. Finally, the Nios II/f processor still showed less improvement, which was only around $5 \%$.

From the results, it can be determined that the obtained saving does not depend on the window size; it only depends on the macro block size and the Nios II processor type. The best improvements are achieved with the FST algorithm and with the Nios II/f processor.

\subsection{Memory system design}

As previously seen, the best advantages of each kind of memory can be utilized to achieve a better design. The design testing here was improved using every possible combination between the selected memories (on-chip and SDRAM) with two different video sequences. The 


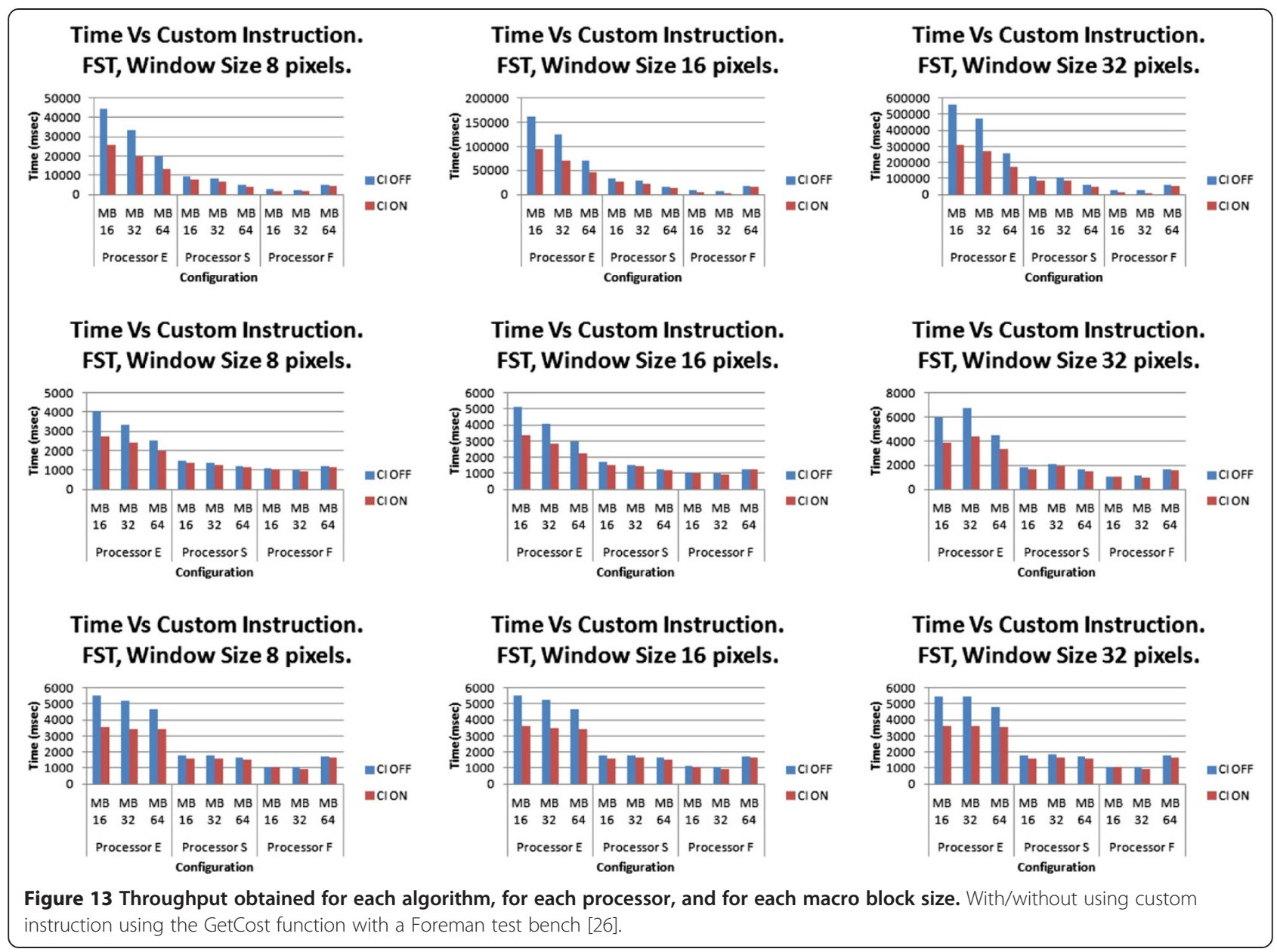

on-chip memory was chosen because it is the fastest available memory on the FPGA and SDRAM due to its large capacity and its lower cost with a good performance. The window size was fixed to 32 pixels and a macro block size of 16 pixels to obtain a viable comparison of the achieved results in each one of the tested algorithms (FST, 2DLOG, and TSST). Presented in Figures 15, 16, 17 are the combinations of memory which obtained a valid design and produced a correct program output in our testing platform, (Altera DE2 board) [27], which incorporates a chip Cyclone II EP2C35F672C6 [28].

Table 6 shows the exact memory system design achieved for each performance. Each one of the possible memories that could be chosen by the designer is shown, as well as which kind of memory (on-chip vs SDRAM) of the chosen types was used.

Configuration types 2 and 3 (in italics) release the better performance since program memory is allocated using on-chip memory.

The second-best configuration groups are designs 6 to 8 , where the Stack is configured to be on-chip memory, and designs 13 to 16 , where the main characteristic in
Stack is configured to on-chip memory. The baseline case (number 1) was considered using SDRAM in every single parameter of the microprocessor design.

\section{Final results: custom instructions and memory choice}

According to the two previous approaches, the embedded system was built by putting them together, in order to enhance the performance results. All possible memory configurations were tested between on-chip and SDRAM memories in order to present the entire scope of possibilities running the three presented algorithms, including (or not) the use of the designed custom instruction, instead of the source GetCost function in every available Nios II processor (E, S, and F). In Table 7, an overview of the FPGA used resources is presented for each one of the possible FPGA configurations, which provide all the possibilities for implementing all of the tested designs.

Regarding Table 6, the FPGA configurations are ordered as follows: the first four rows correspond to the Nios II processor 'e,' the following four rows correspond to the Nios processor 's', and the last four rows correspond 


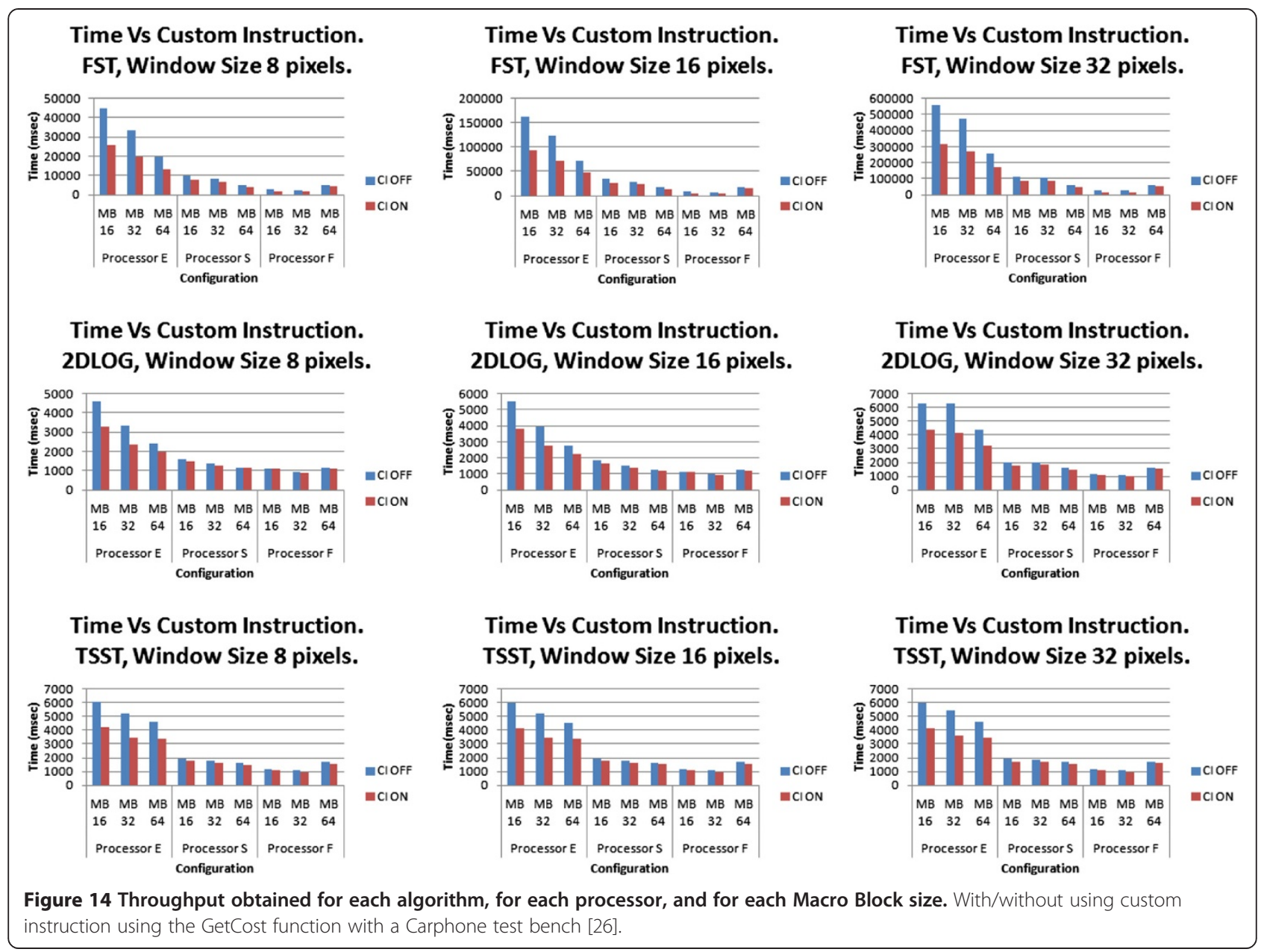

Table 4 Achieved improvements for the Foreman test bench [26]

\begin{tabular}{|c|c|c|c|c|c|c|c|c|c|}
\hline \multirow[t]{3}{*}{ Technique } & \multicolumn{9}{|c|}{ Microprocessor } \\
\hline & \multicolumn{3}{|c|}{ Nios II/e } & \multicolumn{3}{|c|}{ Nios II/s } & \multicolumn{3}{|c|}{ Nios II/f } \\
\hline & MB 16 & MB 32 & MB 64 & MB 16 & MB 32 & MB 64 & MB 16 & MB 32 & MB 64 \\
\hline \multicolumn{10}{|l|}{ FST } \\
\hline 8 pixels & $41.72 \%$ & $41.88 \%$ & $32.23 \%$ & $20.02 \%$ & $16.73 \%$ & $15.84 \%$ & $39.74 \%$ & $36.22 \%$ & $9.13 \%$ \\
\hline 16 pixels & $42.58 \%$ & $42.90 \%$ & $33.29 \%$ & $21.08 \%$ & $18.86 \%$ & $17.91 \%$ & $50.22 \%$ & $44.96 \%$ & $11.15 \%$ \\
\hline 32 pixels & $44.23 \%$ & $43.21 \%$ & $33.62 \%$ & $21.61 \%$ & $18.99 \%$ & $18.42 \%$ & $54.00 \%$ & $53.85 \%$ & $11.49 \%$ \\
\hline \multicolumn{10}{|l|}{ 2DLOG } \\
\hline 8 pixels & $32.35 \%$ & $27.63 \%$ & $20.63 \%$ & $8.78 \%$ & $7.25 \%$ & $4.20 \%$ & $6.36 \%$ & $5.15 \%$ & $4.13 \%$ \\
\hline 16 pixels & $34.05 \%$ & $30.05 \%$ & $23.31 \%$ & $11.63 \%$ & $6.54 \%$ & $3.94 \%$ & $4.63 \%$ & $8.00 \%$ & $4.65 \%$ \\
\hline 32 pixels & $35.45 \%$ & $35.46 \%$ & $25.61 \%$ & $10.11 \%$ & $10.33 \%$ & $11.24 \%$ & $5.50 \%$ & $14.91 \%$ & $5.39 \%$ \\
\hline \multicolumn{10}{|l|}{ TSST } \\
\hline 8 pixels & $34.97 \%$ & $33.46 \%$ & $26.18 \%$ & $11.24 \%$ & $10.56 \%$ & $8.98 \%$ & $0.93 \%$ & $11.21 \%$ & $4.71 \%$ \\
\hline 16 pixels & $34.67 \%$ & $33.14 \%$ & $26.50 \%$ & $11.11 \%$ & $9.44 \%$ & $9.52 \%$ & $4.42 \%$ & $11.21 \%$ & $4.12 \%$ \\
\hline 32 pixels & $34.37 \%$ & $33.46 \%$ & $25.79 \%$ & $9.55 \%$ & $10.22 \%$ & $8.77 \%$ & $\sim 0.00 \%$ & $11.21 \%$ & $7.39 \%$ \\
\hline
\end{tabular}


Table 5 Achieved Improvements for the Carphone test-bench [26]

\begin{tabular}{|c|c|c|c|c|c|c|c|c|c|}
\hline \multirow[t]{3}{*}{ Technique } & \multicolumn{9}{|c|}{ Microprocessor } \\
\hline & \multicolumn{3}{|c|}{ Nios II/e } & \multicolumn{3}{|c|}{ Nios II/s } & \multicolumn{3}{|c|}{ Nios II/f } \\
\hline & M B16 & MB 32 & MB 64 & MB 16 & MB 32 & MB 64 & MB 16 & MB 32 & MB 64 \\
\hline \multicolumn{10}{|l|}{ FST } \\
\hline 8 pixels & $42.43 \%$ & $41.76 \%$ & $31.72 \%$ & $19.12 \%$ & $17.00 \%$ & $15.67 \%$ & $40.39 \%$ & $35.83 \%$ & $9.87 \%$ \\
\hline 16 pixels & $43.58 \%$ & $42.87 \%$ & $33.24 \%$ & $21.02 \%$ & $18.57 \%$ & $17.74 \%$ & $51.73 \%$ & $49.03 \%$ & $10.98 \%$ \\
\hline 32 pixels & $43.91 \%$ & $43.17 \%$ & $33.69 \%$ & $21.44 \%$ & $18.99 \%$ & $18.48 \%$ & $56.01 \%$ & $53.73 \%$ & $11.53 \%$ \\
\hline \multicolumn{10}{|l|}{ 2DLOG } \\
\hline 8 pixels & $28.76 \%$ & $28.53 \%$ & $17.92 \%$ & $6.79 \%$ & $7.91 \%$ & $\sim 0.00 \%$ & $0.92 \%$ & $5.26 \%$ & $1.75 \%$ \\
\hline 16 pixels & $31.65 \%$ & $30.63 \%$ & $20.22 \%$ & $10.44 \%$ & $7.84 \%$ & $5.56 \%$ & $0.88 \%$ & $6.06 \%$ & $3.17 \%$ \\
\hline 32 pixels & $31.05 \%$ & $34.76 \%$ & $26.27 \%$ & $10.15 \%$ & $9.36 \%$ & $7.55 \%$ & $5.13 \%$ & $10.81 \%$ & $4.94 \%$ \\
\hline \multicolumn{10}{|l|}{ TSST } \\
\hline 8 pixels & $30.51 \%$ & $33.65 \%$ & $27.02 \%$ & $9.84 \%$ & $8.94 \%$ & $8.54 \%$ & $8.47 \%$ & $10.38 \%$ & $5.39 \%$ \\
\hline 16 pixels & $30.99 \%$ & $33.85 \%$ & $25.61 \%$ & $10.77 \%$ & $10.11 \%$ & $7.32 \%$ & $6.72 \%$ & $11.82 \%$ & $5.39 \%$ \\
\hline 32 pixels & $30.92 \%$ & $33.52 \%$ & $25.43 \%$ & $10.42 \%$ & $10.22 \%$ & $7.78 \%$ & $9.24 \%$ & $10.19 \%$ & $5.88 \%$ \\
\hline
\end{tabular}

to the Nios 'f' processor. Inside each processor, the group of four rows were divided into two groups of two rows. The first two rows corresponded to the FPGA configuration without custom instruction, and the last two rows corresponded to the FPGA configuration using the custom instruction. Looking at every pair of rows, it can be seen that the first one contains the chip vectors (the reset vector and the exception vector), which were allocated into the on-chip memory, and the second one allocated them into the SDRAM memory.

In Figures 18, 19, and 20, at-a-glance results were obtained running FST, 2DLOG, and TSST, using a macro block size of 16 pixels.

It can be seen from the charts that, as with the previous eight designs, a totally descriptive set can be achieved of the memory system designs shown. For this reason, results are presented for macro block sizes of 32 and 64 pixels using only the eight previous memory system designs. In Figures 21, 22, and 23, the results obtained running FST, 2DLOG, and TSST using a macro block size of 32 pixels can be seen.
Figures 24, 25, and 26 show results obtained running FST, 2DLOG, and TSST fixing the macro block size to 64 pixels.

By examining the above charts some conclusions can be obtained regarding the tests used without custom instructions.

Regarding the Nios II processor 'e', it can be seen that designs are divided into three families, regardless of the macro block size. The first family is formed by designs $1,4,5,9,10,11$, and 12, which have the Text program and the Stack allocated into the SDRAM memory. The second family is formed by designs 2 and 3, which are different because of the storage of the Text program in the on-chip memory, though the Stack is stored in the SDRAM. The third family is formed by designs $6,7,8$, $13,14,15$, and 16, which store the Text program in SDRAM but the Stack into the on-chip memory. As is evident, the second family is the fastest, followed by the third family, and finally, by the first one.

Regarding the Nios II processor 's', it can be seen that designs are divided into two families, regardless of the

Time Vs Memory System Design.

FST, Window Size 32 pixels, Macro Block 16 pixels.

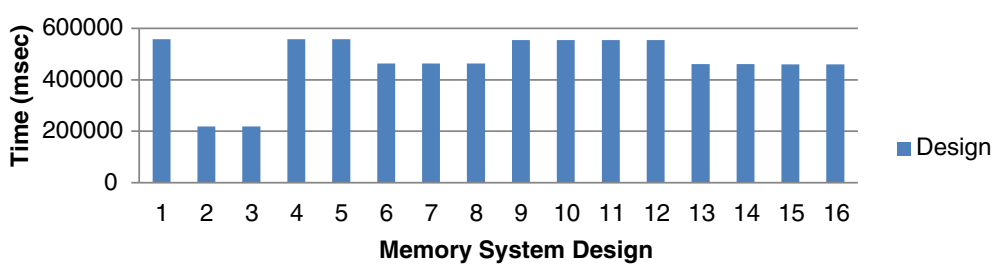

Figure 15 Throughput obtained for each memory system design using FST. 
Time Vs Memory System Design.

2DLOG, Window Size 32 pixels, Macro Block 16 pixels.

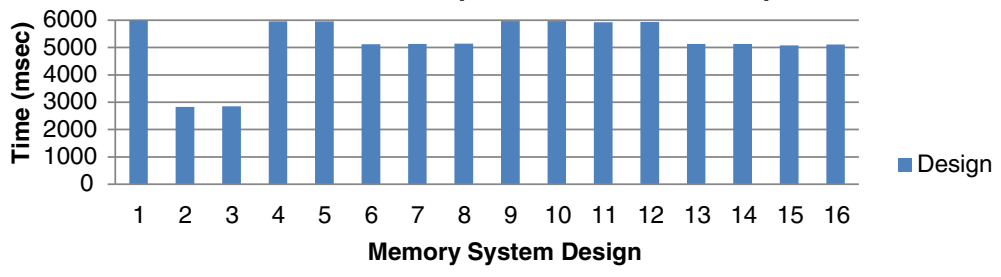

Figure 16 Throughput obtained for each memory system design using 2DLOG.

macro block size. The first one is formed by designs 1 , $2,3,4,5,9,10,11$, and 12 , which are different from the second family, where the Stack is stored in the SDRAM. The second family, and the fastest, is formed by designs $6,7,8,13,14,15$, and 16 , where the Stack is put into the on-chip memory.

Regarding the Nios II processor 'f', it can be seen that all the designs produce a similar throughput using macro block with sizes of 16 and 32 pixels, but by using a macro block with a size of 64 pixels, designs are divided into two families that correspond with the two families described when using the Nios II processor 's.'

What follows are the uses of custom instruction and descriptions of the improvements, depending on the algorithm.

Focusing on the FST algorithm, it can be deduced that the use of custom instructions with the Nios II processor 'e' reduce by nearly $50 \%$, on average, the time spent by the algorithm in every family, particularly regarding the design and the macro block size, except when using a macro block size of 64 pixels, where a slightly lower reduction was achieved, which is around $45 \%$ on average. Using the Nios II processor 's', the use of the custom instruction only depends on the design family. For this reason, the first family allows a profit between $30 \%$ and $35 \%$, but for the second family, an improvement of nearly $50 \%$ was obtained. Finally, with the use of custom instructions in the Nios II processor 'f,' an improvement of around 55\% was achieved in every design when using macro block sizes of 16 and 32 pixels; but, when using a macro block size of 64 pixels, an improvement around $20 \%$ was achieved.

Focusing on the 2DLOG algorithm, it can be concluded that the use of custom instruction in the Nios II processor 'e' has an improvement rate of around 35\% for the first design family, an improvement of $15 \%$ in the execution time for the second family, and for the third, a profit between $30 \%$ and 35\% was obtained. Looking at the Nios II processor 's', a profit between $10 \%$ and $15 \%$ was achieved using custom instructions, regardless of the particular design, although using custom instruction with the Nios II processor 'f' netted an improvement of around $10 \%$ in every design.

Focusing on the TSST algorithm, a profit between 25\% and $35 \%$ was obtained using custom instruction with the Nios II processor ' $e$ ' for the first design family, an improvement of $10 \%$ for the second family, and a reduction between $30 \%$ and $35 \%$ for the third family regarding the execution time. Using the Nios II processor 's' and custom instructions, an improvement between $10 \%$ and $15 \%$ was achieved, regardless of any particular design or macro block size. Finally, using the Nios II processor ' $\mathrm{f}$ ' and custom instructions, a profit between $0 \%$ and $10 \%$ was obtained in every design, regardless of the fixed macro block size.

Gathering all the previous results from the experiments, the following conclusions were made: regarding the Nios II processor 'e', the fastest family is the second family, which netted a profit, on average, of nearly $75 \%$ (for FST MB 16, 32, and 64); around 60\% (for 2DLOG

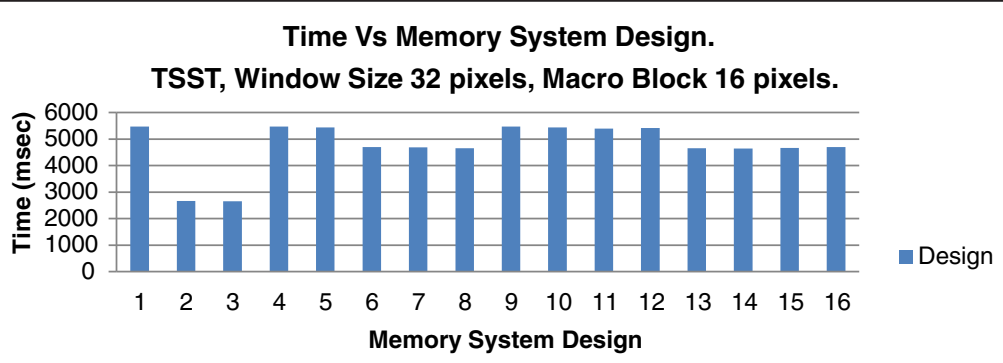

Figure 17 Throughput obtained for each memory system design using TSST. 
Table 6 Memory system design configuration

\begin{tabular}{|c|c|c|c|c|c|c|c|}
\hline \multirow[t]{2}{*}{ Design } & \multicolumn{7}{|c|}{ Memories } \\
\hline & $\begin{array}{l}\text { Processor reset } \\
\text { vector }\end{array}$ & $\begin{array}{l}\text { Processor exception } \\
\text { vector }\end{array}$ & Stack & Heap & $\begin{array}{l}\text { Read/write data } \\
\text { (.rwdata) }\end{array}$ & $\begin{array}{l}\text { Read only data } \\
\text { (.rodata) }\end{array}$ & Program (.text) \\
\hline 1 & SDRAM & SDRAM & SDRAM & SDRAM & SDRAM & SDRAM & SDRAM \\
\hline 2 & SDRAM & SDRAM & SDRAM & SDRAM & SDRAM & SDRAM & On-Chip \\
\hline 3 & SDRAM & SDRAM & SDRAM & SDRAM & SDRAM & On-Chip & On-Chip \\
\hline 4 & SDRAM & SDRAM & SDRAM & SDRAM & On-Chip & SDRAM & SDRAM \\
\hline 5 & SDRAM & SDRAM & SDRAM & SDRAM & On-Chip & On-Chip & SDRAM \\
\hline 6 & SDRAM & SDRAM & On-Chip & SDRAM & SDRAM & SDRAM & SDRAM \\
\hline 7 & SDRAM & SDRAM & On-Chip & SDRAM & On-Chip & SDRAM & SDRAM \\
\hline 8 & SDRAM & SDRAM & On-Chip & SDRAM & On-Chip & On-Chip & SDRAM \\
\hline 9 & On-chip & On-chip & SDRAM & SDRAM & SDRAM & SDRAM & SDRAM \\
\hline 10 & On-chip & On-chip & SDRAM & SDRAM & SDRAM & On-chip & SDRAM \\
\hline 11 & On-chip & On-chip & SDRAM & SDRAM & On-chip & SDRAM & SDRAM \\
\hline 12 & On-chip & On-chip & SDRAM & SDRAM & On-chip & On-Chip & SDRAM \\
\hline 13 & On-chip & On-chip & On-chip & SDRAM & SDRAM & SDRAM & SDRAM \\
\hline 14 & On-chip & On-chip & On-chip & SDRAM & SDRAM & On-chip & SDRAM \\
\hline 15 & On-chip & On-chip & On-chip & SDRAM & On-chip & SDRAM & SDRAM \\
\hline 16 & On-chip & On-chip & On-chip & SDRAM & On-chip & On-chip & SDRAM \\
\hline
\end{tabular}

MB 16, 32, and 64); and nearly 60\% (for TSST MB 16, 32 , and 64), depending on the algorithm. Using the Nios II processor 's', the fastest family was shown to be the second family, too, reducing the execution time, on average, nearly $75 \%$ (for FST MB 16, 32, and 64); around $20 \%$ (for 2 DLOG MB 16, 32, and 64); and nearly $40 \%$ (for TSST MB 16, 32, and 64) in every algorithm. Finally, using the Nios II processor ' $\mathrm{f}$ ' for the FST, an improvement was achieved, on average, of around 55\% (for FST MB 16, 32, and 64); around 40\% (for 2DLOG MB 16, 32, and 64); and nearly 20\% (TSST MB 16, 32, and 64) on each algorithm.

To contrast the experiments, the significant set of the memory system designs previously described (1 to 8 ) was tested using the Carphone test bench [26], also previously described.

Table 7 FPGA used resources

\begin{tabular}{|c|c|c|c|c|c|c|c|c|c|c|c|c|}
\hline \multicolumn{13}{|c|}{ FPGA resources } \\
\hline $\begin{array}{l}\text { Logic } \\
\text { cells }\end{array}$ & $\begin{array}{c}\text { Dedicated logic } \\
\text { registers }\end{array}$ & $\begin{array}{c}\mathrm{I} / \mathrm{O} \\
\text { registers }\end{array}$ & $\begin{array}{l}\text { Memory } \\
\text { bits }\end{array}$ & M4Ks & $\begin{array}{c}\text { DSP } \\
\text { elements }\end{array}$ & $\begin{array}{l}\text { DSP } \\
9 \times 9\end{array}$ & $\begin{array}{c}\text { DSP } \\
18 \times 18\end{array}$ & Pins & $\begin{array}{l}\text { Virtual } \\
\text { pins }\end{array}$ & $\begin{array}{l}\text { LUT-only } \\
\text { LCs }\end{array}$ & $\begin{array}{c}\text { Register-only } \\
\text { LCs }\end{array}$ & $\begin{array}{c}\text { LUT/register } \\
\text { LCs }\end{array}$ \\
\hline $2202(1)$ & $1050(0)$ & $52(52)$ & 306,176 & 78 & 0 & 0 & 0 & 56 & 0 & $1,152(1)$ & $148(0)$ & $902(0)$ \\
\hline 2198(1) & $1050(0)$ & $52(52)$ & 306,176 & 78 & 0 & 0 & 0 & 56 & 0 & $1,148(1)$ & $148(0)$ & $902(0)$ \\
\hline $2352(1)$ & $1051(0)$ & $52(52)$ & 306,176 & 78 & 0 & 0 & 0 & 56 & 0 & $1,301(1)$ & $148(0)$ & $903(0)$ \\
\hline $2348(1)$ & $1051(0)$ & $52(52)$ & 306,176 & 78 & 0 & 0 & 0 & 56 & 0 & $1,297(1)$ & $146(0)$ & $905(0)$ \\
\hline $3152(1)$ & 1755(0) & $52(52)$ & 341,632 & 87 & 4 & 0 & 2 & 56 & 0 & $1,397(1)$ & 228(0) & $1,527(0)$ \\
\hline $3150(1)$ & 1755(0) & $52(52)$ & 341,632 & 87 & 4 & 0 & 2 & 56 & 0 & $1,395(1)$ & $225(0)$ & $1,530(0)$ \\
\hline $3284(1)$ & $1756(0)$ & $52(52)$ & 341,632 & 87 & 4 & 0 & 2 & 56 & 0 & $1,528(1)$ & $223(0)$ & $1,533(0)$ \\
\hline $3285(1)$ & $1756(0)$ & $52(52)$ & 341,632 & 87 & 4 & 0 & 2 & 56 & 0 & $1,529(1)$ & $223(0)$ & $1,533(0)$ \\
\hline $3868(1)$ & 2175(0) & $52(52)$ & 377,088 & 98 & 4 & 0 & 2 & 56 & 0 & $1,693(1)$ & $363(0)$ & $1,812(0)$ \\
\hline $3858(1)$ & $2175(0)$ & $52(52)$ & 377,088 & 98 & 4 & 0 & 2 & 56 & 0 & $1,683(1)$ & $360(0)$ & $1,815(0)$ \\
\hline 3973(1) & 2178(0) & $52(52)$ & 377,088 & 98 & 4 & 0 & 2 & 56 & 0 & $1,795(1)$ & $360(0)$ & $1,818(0)$ \\
\hline $3968(1)$ & $2178(0)$ & $52(52)$ & 377,088 & 98 & 4 & 0 & 2 & 56 & 0 & $1,790(1)$ & $360(0)$ & $1,818(0)$ \\
\hline
\end{tabular}


Time Vs Design.

FST, Window Size 32 pixels, Macro Block 16 pixels.

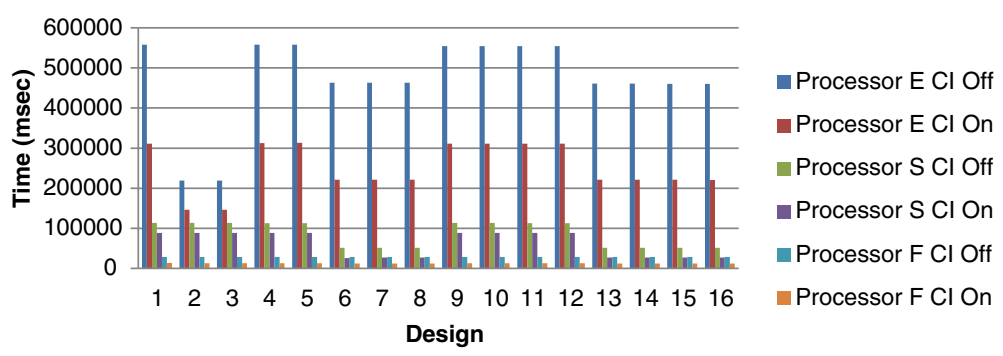

Figure 18 Performance of final throughput (Custom instruction + Memory optimization) for FST-algorithm Nios II processor. ('economic', 'standard', and 'fast').

Time Vs Design.

2DLOG, Window Size 32 pixels, Macro Block 16 pixels.

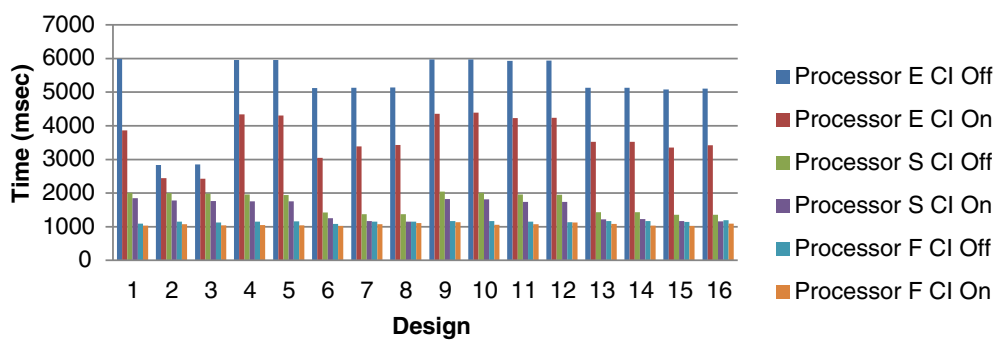

Figure 19 Performance of final throughput (Custom instruction + Memory Optimization) for 2DLOG-algorithm Nios II processor. ('economic', 'standard', and 'fast').

Time Vs Design.

TSST, Window Size 32 pixels, Macro Block 16 pixels.

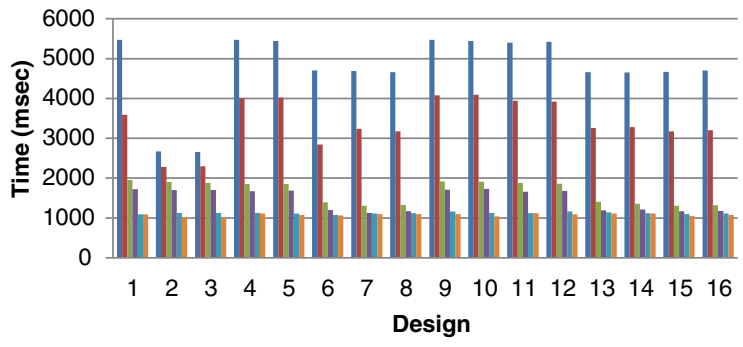

- Processor E Cl Off - Processor E Cl On Processor S Cl Off Processor S ClOn Processor F Cl Off - Processor $\mathrm{F} \mathrm{ClOn}$

Figure 20 Performance of final throughput (Custom instruction + Memory optimization) for TSST-algorithm Nios II processor. ('economic', 'standard', and 'fast'). 
Time Vs Design.

FST, Window Size 32 pixels, Macro Block 32 pixels.

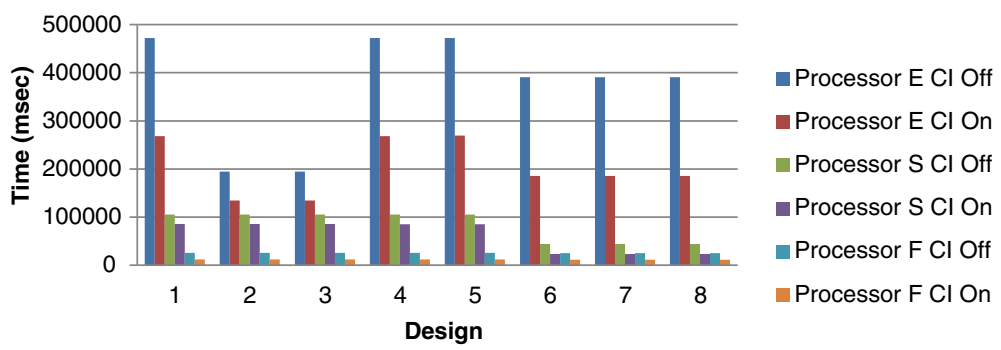

Figure 21 Performance of final throughput (Custom instruction + Memory optimization) for FST-algorithm Nios II processor. ('economic', 'standard', and 'fast').

Time Vs Design.

2DLOG, Window Size 32 pixels, Macro Block 32 pixels.

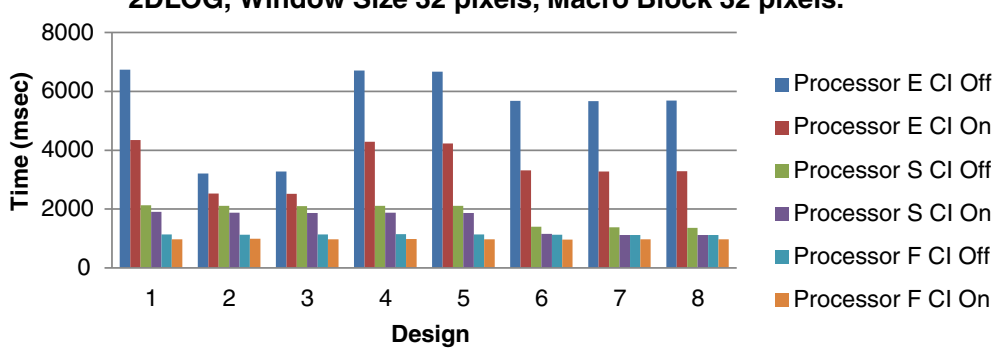

Figure 22 Performance of the final throughput (Custom instruction + Memory optimization) for 2DLOG-algorithm Nios II processor. ('economic', 'standard', and 'fast').

Time Vs Design.

TSST, Window Size 32 pixels, Macro Block 32 pixels.

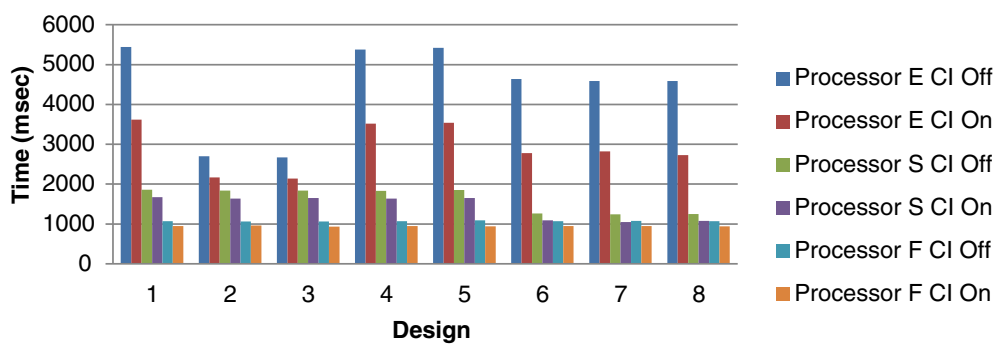

Figure 23 Performance of final throughput (Custom instruction + Memory optimization) for TSST-algorithm Nios II processor. ('economic', 'standard', and 'fast'). 
Time Vs Design.

FST, Window Size 32 pixels, Macro Block 64 pixels.

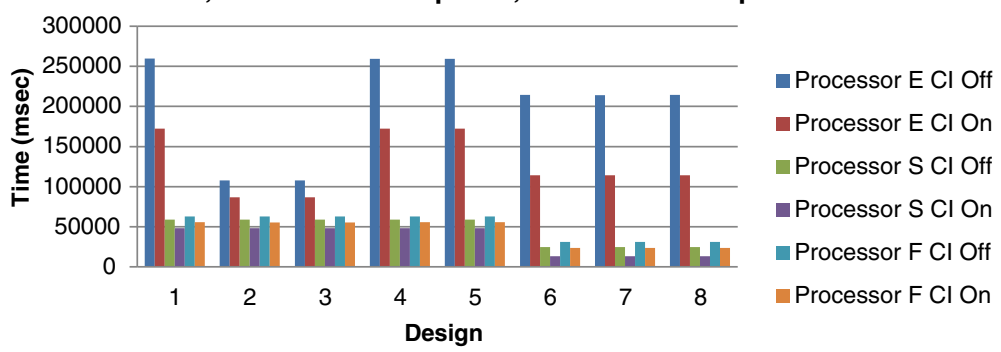

Figure 24 Performance of final throughput (Custom instruction + Memory optimization) for FST-algorithm Nios II processor. ('economic', 'standard', and 'fast').

Time Vs Design.

2DLOG, Window Size 32 pixels, Macro Block 64 pixels.

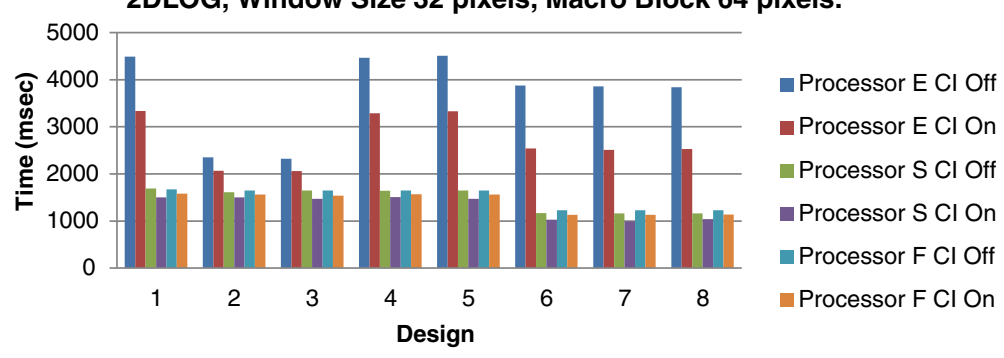

Figure 25 Performance of final throughput (Custom instruction + Memory optimization) for 2DLOG-algorithm Nios II processor. ('economic', 'standard', and 'fast').

Time Vs Design.

TSST, Window Size 32 pixels, Macro Block 64 pixels.

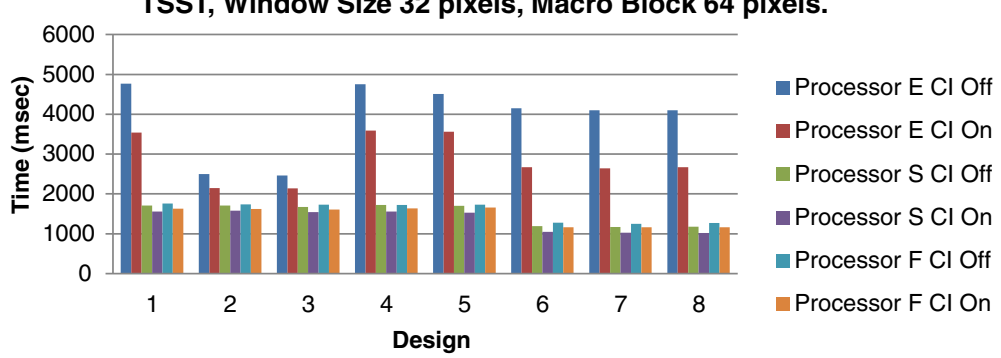

Figure 26 Performance of final throughput (Custom instruction + Memory optimization) for TSST-algorithm Nios II processor. ('economic', 'standard', and 'fast'). 
Time Vs Design.

FST, Window Size 32 pixels, Macro Block 16 pixels.

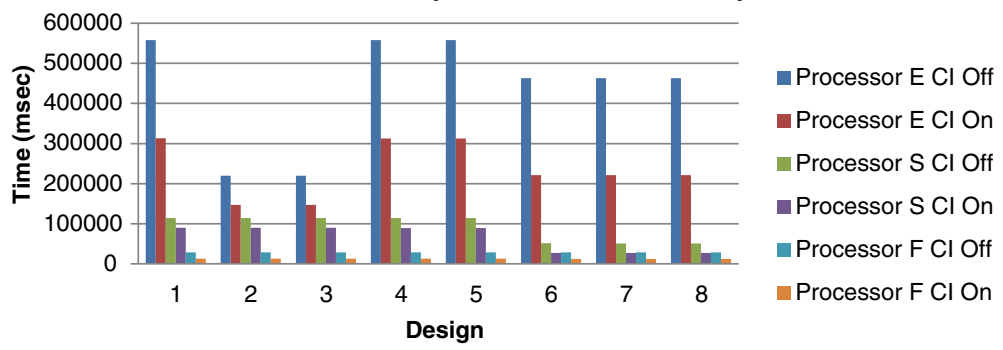

Figure 27 Performance of final throughput (Custom instruction + Memory optimization) for FST-algorithm Nios II processor. ('economic', 'standard', and 'fast').

Time Vs Design.

2DLOG, Window Size 32 pixels, Macro Block 16 pixels.

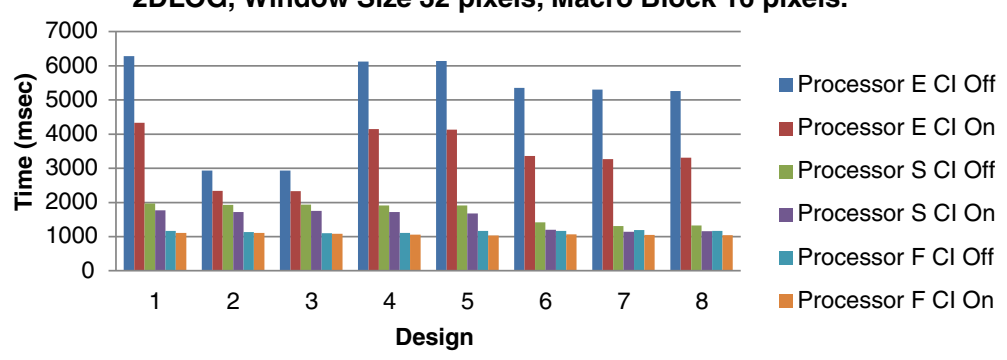

Figure 28 Performance of final throughput (Custom instruction + Memory optimization) for 2DLOG-algorithm Nios II processor. ('economic', 'standard', and 'fast').

Time Vs Design.

TSST, Window Size 32 pixels, Macro Block 16 pixels.

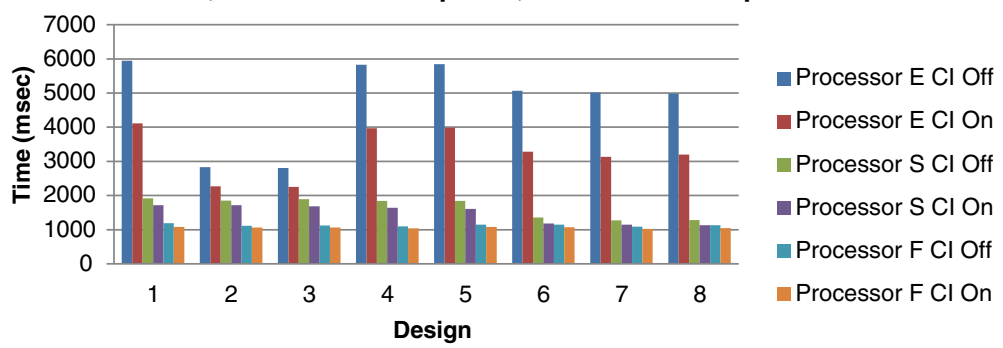

Figure 29 Performance of final throughput (Custom instruction + Memory optimization) for TSST-algorithm Nios II processor. ('economic', 'standard', and 'fast'). 
Time Vs Design.

FST, Window Size 32 pixels, Macro Block 32 pixels.

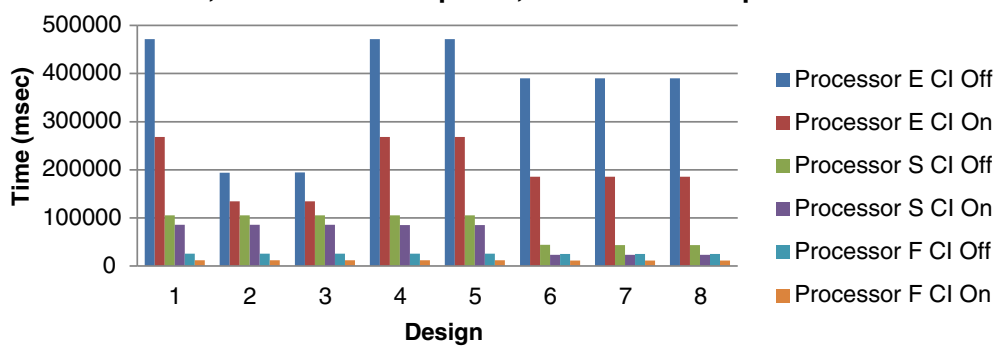

Figure 30 Performance of final throughput (Custom instruction + Memory optimization) for full-algorithm Nios II processor. ('economic', 'standard', and 'fast').

Time Vs Design.

2DLOG, Window Size 32 pixels, Macro Block 32 pixels.

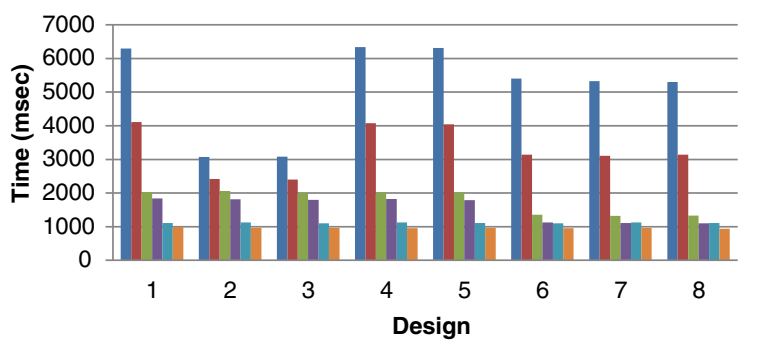

Processor E Cl Off

- Processor E Cl On

- Processor S Cl Off

- Processor S CI On

- Processor F Cl Off

Processor $\mathrm{F} \mathrm{Cl} \mathrm{On}$

Figure 31 Performance of final throughput (Custom instruction + Memory optimization) for 2DLOG-algorithm Nios II processor. ('economic', 'standard', and 'fast').

Time Vs Design.

TSST, Window Size 32 pixels, Macro Block 32 pixels.

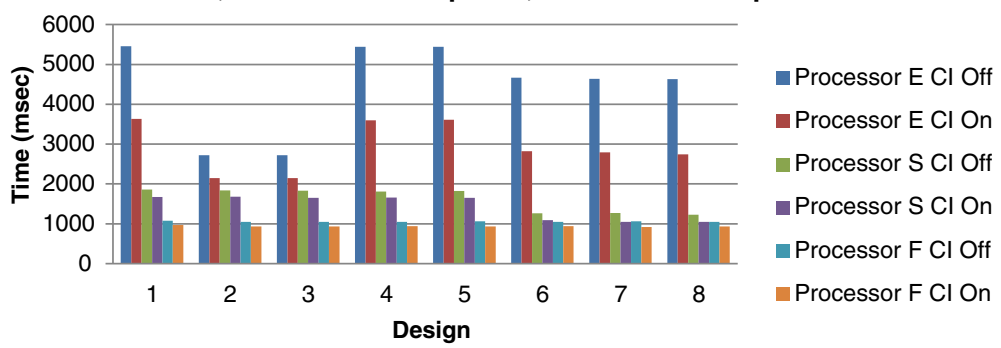

Figure 32 Performance of final throughput (Custom instruction + Memory optimization) for TSST-algorithm Nios II processor. ('economic', 'standard', and 'fast'). 
Time Vs Design.

FST, Window Size 32 pixels, Macro Block 64 pixels.

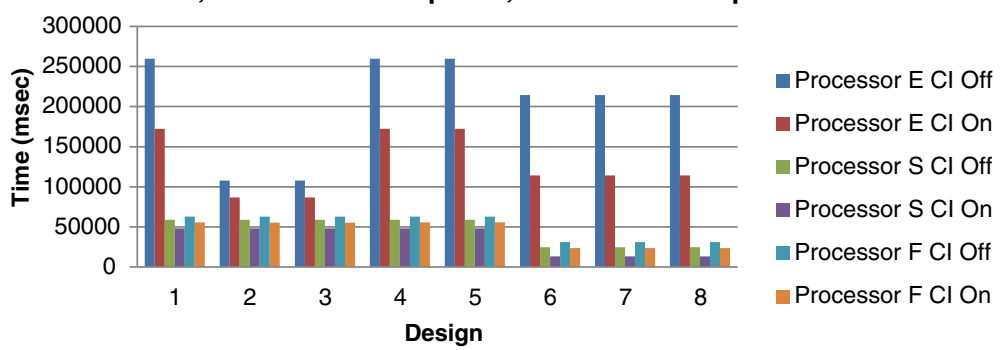

Figure 33 Performance of final throughput (Custom instruction + Memory optimization) for full-algorithm Nios II processor. ('economic', 'standard', and 'fast').

Time Vs Design.

2DLOG, Window Size 32 pixels, Macro Block 64 pixels.

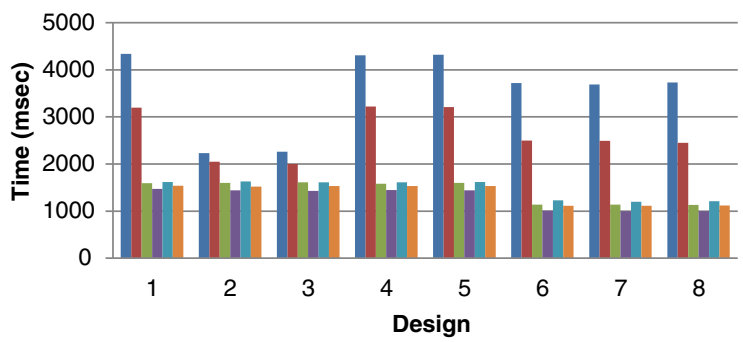

- Processor E Cl Off

- Processor E CIOn

- Processor $\mathrm{S} \mathrm{Cl}$ Off

- Processor S Cl On

- Processor $\mathrm{F} \mathrm{Cl} \mathrm{Off}$

- Processor $\mathrm{F} \mathrm{Cl}$ On

Figure 34 Performance of final throughput (Custom instruction + Memory optimization) for 2DLOG-algorithm Nios II processor. ('economic', 'standard', and 'fast').

Time Vs Design.

TSST, Window Size 32 pixels, Macro Block 64 pixels.

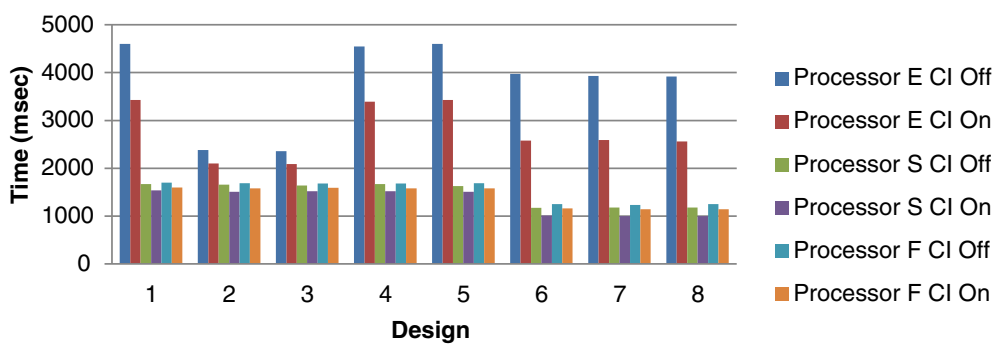

Figure 35 Performance of final throughput (Custom instruction + Memory optimization) for TSST-algorithm Nios II processor. ('economic', 'standard', and 'fast'). 
Figures 27, 28, and 29 show the results obtained running FST, 2DLOG, and TSST fixing the macro block size to 16 pixels.

Figures 30, 31, and 32 show the obtained results running FST, 2DLOG, and TSST fixing the macro block size to 32 pixels.

Finally, Figures 33, 34, and 35 show the obtained results running FST, 2DLOG, and TSST by fixing the macro block size to 64 pixels.

\section{Conclusions}

In this paper, the problem of the acceleration motion estimation algorithm, widely used for video coding MPEG (H.264/6), was addressed by building an FPGA-based low-cost embedded system, the Altera DE2 platform, and customizing the well-known soft-core microprocessor Nios II. The technique developed here has been separately evaluated using a custom instruction paradigm through a combinational instruction and the efficient combination of on-chip memory and SDRAM regarding the reset vector, exception vector, stack, heap, read/write data (.rwdata), read only data (.rodata), and program text (.text) in the design. A combination of two methods was then developed to build the final embedded system. With the use of custom instructions, an improvement was reached of $23 \%$, on average, but close to a $55 \%$ improvement, in the best case, which supposes a great amount of savings in time spent for execution. With a better use of the memory types available in the design, an improvement of $61 \%$ was achieved in the execution time. With the combination of both techniques, an improvement of $75 \%$ was achieved against the base case.

\section{Competing interests}

The authors declare that they have no competing interests.

\section{Acknowledgments}

The authors would like to thank the Altera Company for the provided hardware and software under the University programs. The authors would like to thank Professor Uwe Meyer-Bäse from Florida State University for his help and support regarding digital signal processing with FPGAs. This work has been partially supported by Spanish Projects TIN 2008/508 and TIN 2012/32180.

Received: 15 February 2013 Accepted: 29 May 2013

Published: 16 June 2013

\section{References}

1. D Marpe, T Wiegand, GJ Sullivan, The H.264/MPEG4 advanced video coding standard and its applications. IEEE Commun Mag 44, 134-143 (2006)

2. ITU-T Recommendation H.264 (draft), International standard for advanced video coding (ITU-T, Geneva, 2003)

3. ITU-T Recommendation H.264 \& ISO/IEC 14496-10 (MPEG-4) AVC, Advance Video Coding for Generic Audiovisual Services (ITU-T, Geneva, 2005)

4. J Konrad, Estimating motion in image sequences. IEEE Signal Process Mag 16, 70-91 (1999)

5. S Kappagantula, K-R Rao, Motion compensated interframes image prediction. IEEE Trans Commun 33, 1011-1015 (1985)

6. C-J Kuo, C-H Yeh, S-F Odeh, Polynomial search algorithms for motion estimation, in Proceedings of the 1999 IEEE International Symposium on Circuits and System (ISCAS'99), vol. 4 (Orlando, 1999), pp. 215-218
7. S Zhu, K-K Ma, A new diamond search algorithm for fast block-matching motion estimation. IEEE Trans Image Process 9, 287-290 (2000)

8. S Zhu, Fast motion estimation algorithms for video coding (Nanyang Technology University, Singapore, M.S. thesis, 1998)

9. F Ayuso, G Botella, C García, M Prieto, F Tirado, GPU-based acceleration of bio-inspired motion estimation model. Concurrency and Computation: Practice and Experience 25, 1037-1056 (2013). doi:10.1002/cpe.2946

10. G Botella, A García, M Rodriguez-Alvarez, E Ros, U Meyer-Bäse, MC Molina, Robust bioinspired architecture for optical-flow computation. IEEE Trans. VLSI Syst. 18(4), 616-629 (2010)

11. C Garcia, G Botella, F Ayuso, M Prieto, F Tirado, Multi-GPU based on multicriteria optimization for motion estimation system. EURASIP JOURNAL on Advances in Signal Processing 2013, 23 (2013)

12. D González, G Botella, U Meyer-Baese, C García, C Sanz, M Prieto-Matías, F Tirado, A Low, Cost Matching Motion Estimation Sensor Based on the NIOS II Microprocessor. Sensors 12, 13126-13149 (2012)

13. T Koga, K linuma, A Hirano, Y lijima, T Ishiguro, Motion compensated interframe coding for video conferencing, in Proc. of the Nat. Telecommunications Conference (New Orleans, LA, 1981), pp. G5.3.1-G5.3.5

14. J-R Jain, A-K Jain, Displacement measurement and its application in interframes image coding. IEEE Trans Commun 29, 1799-1808 (1981)

15. B Liu, A Zaccarin, New fast algorithms for estimation of block motion vectors. IEEE Trans. Circuit. Syst. Video Technol. 3, 148-157 (1993)

16. R Li, B Zeng, M-L Liou, A new three-step search algorithm for block motion estimation. IEEE Trans. Circuit. Syst. Video Technol. 4, 438-422 (1994)

17. Altera, Nios II processor: the world's most versatile embedded processor, (2013). http://www.altera.com/devices/processor/nios2/ni2-index.html. Accessed 10 June 2013

18. P Chu, Embedded SoPC Design with NIOS II Processor and Examples (Wiley, Hoboken, 2012)

19. Altera, Nios II performance benchmarks, (2013). http://www.altera.com/ literature/ds/ds_nios2_perf.pdf. Accessed 10 June 2013

20. Altera, Documentation: Nios processor, (2013). http://www.altera.com/ literature/lit-nio.jsp. Accessed 10 June 2013

21. Arm, ARM: the architecture for the digital world, (2013). http://www.arm. com/products/processors/classic/arm9/. Accessed 10 June 2013

22. Altera, Stratix II FPGA: high performance with great signal integrity, (2013). http://www.altera.com/devices/fpga/stratix-fpgas/stratix-ii/stratix-ii/st2-index. jsp. Accessed 10 June 2013

23. Altera, Hardware acceleration, (2013). http://www.altera.com/devices/ processor/nios2/benefits/performance/ni2-acceleration.html. Accessed 10 June 2013

24. Altera, Nios II custom instruction user guide, (2013). http://www.altera.com/ literature/ug/ug_nios2_custom_instruction.pdf. Accessed 10 June 2013

25. Y Mandravellos, Code::Blocks IDE, (2006). https://launchpad.net/codeblocks. Accessed 10 June 2013

26. C Yushin, CIPR sequences, (2013). http://www.cipr.rpi.edu/resource/ sequences/. Accessed 10 June 2013

27. Altera, DE2 development and education board, (2013). http://www.altera. com/education/univ/materials/boards/de2/unv-de2-board.html. Accessed 10 Feb 2013

28. Altera, Cyclone II FPGAs at cost that rivals ASICs, (2012). http://www.altera. com/devices/fpga/cyclone2/cy2-index.jsp. Accessed 10 June 2013

doi:10.1186/1687-6180-2013-118

Cite this article as: González et al:: Acceleration of block-matching algorithms using a custom instruction-based paradigm on a Nios II microprocessor. EURASIP Journal on Advances in Signal Processing 2013 2013:118. 